\title{
Production of triterpenoids with cell and tissue cultures*
}

\author{
Magdalena Malinowska ${ }^{\bowtie}$, Elżbieta Sikora and Jan Ogonowski \\ Institute of Organic Chemistry and Technology Institute, Faculty of Chemical Engineering and Technology, Cracow University of Technology, \\ Kraków, Poland
}

Triterpenes are group of biologically active compounds which can be found in higher plants. Their main source are plants bark, leaves, twigs, fruits, resins or oils. The biological activity of triterpens is very diversified and many studies have already confirmed the following therapeutic effects: anti-inflammatory, antimicrobial, antiviral, antifungial, immunomodulatory, and hepatoprotective. Synthesis of triterpenes derivates can be performed by chemical or enzymatic reactions, however biotransformation is more specific and eliminates the side products and the molecule alterations. These processes use isolated enzymes or microorganisms. Cell culture in vitro eliminates problems like extract variability as well as instability of the compounds being obtained during the extraction process. What is more, it ensures high reproducibility and optimal regio- and enantioselectivity. The most widely used technique is a classical screening of a series of microbial strains. Studies on triterpene biotransformation give a lot of information about new biologically active compounds and let predict the metabolism of biological compounds. This review presents most important advancements in the metabolic engineering of microorganisms for the production of triterpenoids. Moreover, the review highlights general strategies to obtain rich biochemical diversity of plants by employing the biocatalysts produced by microorganisms or tissue cultures.

Key words: biotransformation, tritepenoids, cell cultures, microorganisms, in vitro

Received: 16 October, 2013; revised: 04 December, 2013; accepted: 04 December, 2013; available on-line: 29 December, 2013

\section{INTRODUCTION}

Triterpenes are a group of biologically active compounds which are present in plant tissues in almost every geographical region in the world. The group consist of approximately 30000 identified compounds (Dzubak et al., 2006; Mufflera et al., 2011). The number of terpenoids that are produced by plants is probably larger than that of any other group of natural compounds. Plants have the ability to produce thousands of chemical variations of a single isoprenoid unit. This group includes essential oils and resins, cytokinins, gibberellins, strigolactones, dolichols, various steroids and carotenoids (Lohr et al., 2012).

In most of these compounds, two or more isoprene units are joined together in a head-to-tail configuration of the carbon atoms, either in open chain or in cyclic systems containing one or more rings (Fig. 1). The main exception to the head-to-tail arrangement involves the formation of artemisia ketone (and related mono- terpenoids), squalene, gossypol, and the carotenoids (Waller, 1970; Chatterjee et al., 2000; Parra et al., 2009; Marienhagen \& Bott, 2013).

The major isoprenoids having important function in plant cells are sterols which control fluidity of the plasma membranes, the long-chain polyprenyl dolichol as sugar-carrier-lipid used in protein glycosylation in eukaryotic endoplasmic reticulum. The primary metabolites of eukaryotic organisms includes also phytol, an isoprenoid whis is part of chlorophylls, tocopherols and phylloquinones, the various carotenoids, that are essential as functional and structural components of the photosynthetic routes. What is more, the medium-chain polyprenyl plastoquinone plays an important role in electron transfer during this process. Moreover, isoprenoids serve as precursors of components of various phytohormones (Lohr et al., 2012).

The biological activity of triterpens is very diverse. Many studies have already confirmed the following therapeutic effects: anti-inflammatory, analgesic, antimicrobial, antiviral, antimycotic, immunomodulatory, and hepatoprotective (Dzubak et al., 2006). What is more, modification of the triterpenes structure to enhance their pharmaceutical application can be efficiently carried out using bioprocesses. As previously mentioned, many triterpenes exhibit significant biological activity, but several triterpenoids show hemolytic and cytostatic properties that can restrict their pharmaceutical use. To overcome these limitations and to expand the range of usable triterpenes, a transformation of the compound by means of chemical or biotechnological techniques is possible. However, biotransformation is used to achieve compounds with an optimal regio- and enantioselectivity (Mufflera et al., 2011). Microbial transformations of triterpenoids have been developed primarily in the last 10 or 15 years to produce new and useful compounds (Parra et al., 2009). Biotransformation methods are used as an alternative route to the traditional chemical synthesis in search of new production routes for chemical, pharmaceutical, and agrochemical compounds (Fu et al., 2013). Advantages of these processes are also associated with the environmentally friendly reaction conditions. Moreover, microbial transformation is often the only way to obtain desired product (Zhanga et al., 2005). Recently, several studies have shown that microbial transformation is a useful tool to improve the structural diversity of naturally occurring triterpenoids (Cheng et al., 2004; Qian et al., 2009). Finding out an optimal organism is the most

e-mail: mmalinowska@chemia.pk.edu.pl

*Presented at the 5th Central European Congress of Life Sciences „EUROBIOTECH 2013", Kraków, Poland. 


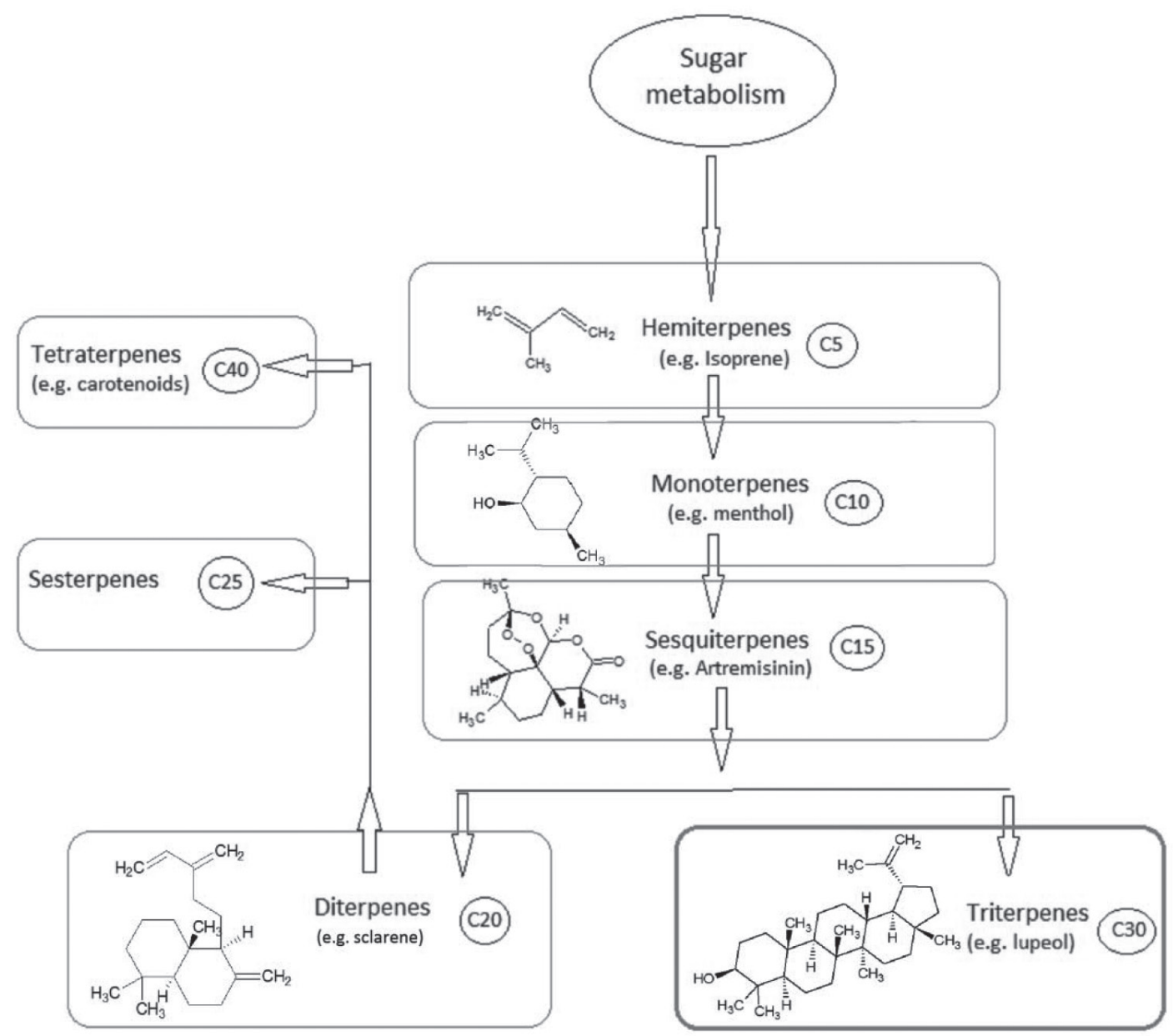

Figure 1. Schematic overview of plant biosynthetic routes of the terpenes (Waller, 1970)

important step in mentioned technology. Comprehensive review on microbial transformation of triterpenoids has been already published (Parra et al., 2009). Much of the rich chemical diversity of natural compounds arises from a limited number of chemical scaffolds (e.g. terpene and polyketide structures), which are modified by specific types of chemical substitutions (hydroxylation, glycosylation, acylation, O-methylation) brought about by substrate- and/or proper enzymes. The way these substances are formed is explained by concerted molecular, genomic and genetic approaches (Dixon, 2005). Such approach provides sufficient quantities of the desired plant natural products from inexpensive renewable resources. Because biological raw materials must be derived from well-known plant sources with reproducible content levels of the drug or its precursors, domestic cultivation of the plant sources has several advantages compared to harvesting from the wild. These advantages minimize the risk of misidentification, genetic and phenotypic variability, extract variability and instability, toxic compounds, and contaminants (Canter et al., 2005). This review, presents a general information concerning processes used for the production of pharmaceutically relevant triterpenes. Moreover, examples of triterpene production by cell and tissue biotransformation and the corresponding analytical techniques, to obtain bioactive compounds from precursor molecules are covered.<smiles>CC1(C)CC[C@]2(C(=O)O)CC[C@]3(C)C(=CCC4C5(C)CC[C@H](O)C(C)(C)C5CCC43C)C2C1</smiles>

oleanolic acid<smiles>CC12CC[C@H](O)C(C)(C)C1CCC1(C)C2CC=C2C3C[C@@](C)(CO)CC[C@@]3(C(=O)O)CC[C@]21C</smiles>

queretaroic acid<smiles>CC1(C)C(O)CC[C@@]2(C)C1CC(O)C1(C)C2CC=C2C3C[C@@](C)(CO)CC[C@@]3(C(=O)O)CC[C@]21C</smiles>

cmpd1

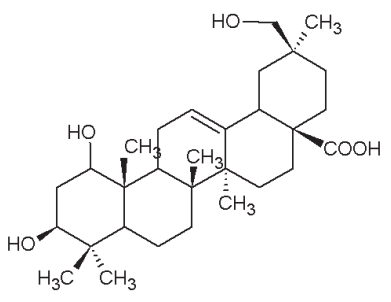

cmpd 2

Figure 2. Metabolites from biotransformation of oleanolic acid with Rhizomucor miehei 


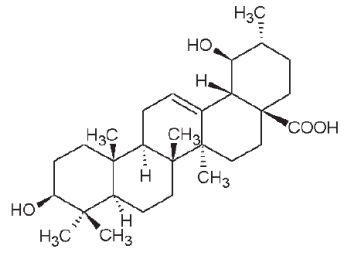

ursolic acid

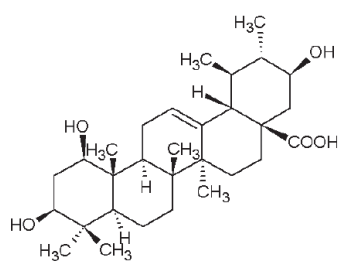

cmpd 5

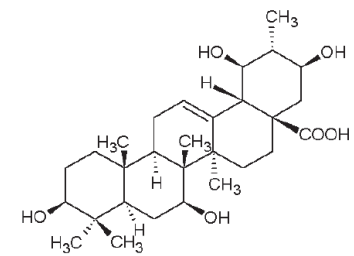

cmpd 3

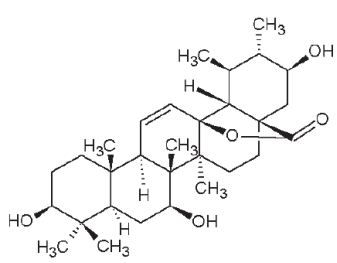

cmpd 6

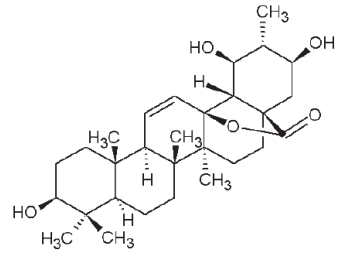

cmpd 4

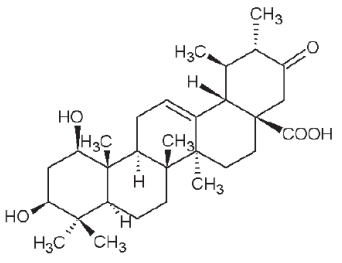

cmpd 7

Figure 3. Biotransformation of ursolic acid by Syncephalastrum racemosum

\section{MATERIALS AND METHODS OF TRITERPENOIDS BIOTRANSFORMATION}

The most widely used technique is still classical screening of a series of microbial strains. The proper cell suspension is prepared in sterile conditions (Chatterjee et al., 2000; Parra et al., 2009; Fu et al., 2013; Mariengagen \& Bott, 2013). The substrate can be micro-emulsified in mixtures of detergents and organic solvents. Proper cells are cultivated in flasks containing a culture medium (i.e. D-(-)-glucose, malt and yeast extract) (Cheng et al., 2004; Canter et al., 2005; Zhanga et al. 2005; Chen et al., 2009; Qian et al., 2009). When the culture medium is separated, proper triterpene dissolved in a nontoxic solvent is added to the flask (Carvalho et al., 2010; Leipolda et al., 2010; Parshikov et al., 2012; Baratto et al., 2013). After few days the broth is filtered and extracted. The medium culture is then sonicated (Bastos et al., 2007; Gallo et al., 2009). The organic fractions are combined, dried and analyzed by Thin Layer Chromatography (TLC). The crude extracts are then fractionated using column, flash or circular chromatography (Charlwood \& Rhodes, 1990; Dornenburg, 2004; Lee et al., 2004; Vanisree et al., 2004; Pavlov et al., 2007). The most common analytical methods used for triterpens identification are TLC (Thin Layer Chromatography), NMR (Nuclear Magnetic Resonance) and IR (Infra Red spectroscopy), HR-MS (High Resolution Mass Spectra) and Polarimetry (Parra et al., 2009).

\section{MICROBIAL TRANSFORMATION OF TRITERPENOIDS}

Studies on triterpene biotransformation give various information on newly syntethised biologically active compounds. Moreover, they let predict the metabolism of biological compounds. Oleanolic acid, (Fig. 2)

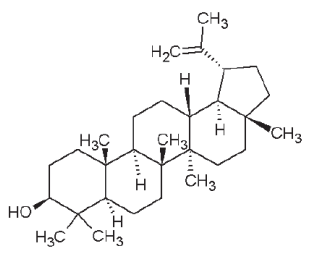

lupeol

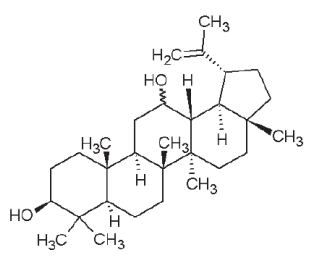

cmpd 12

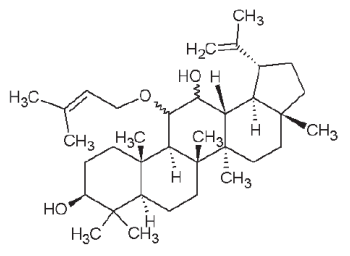

cmpd 13

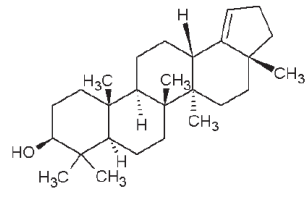

cmpd 14

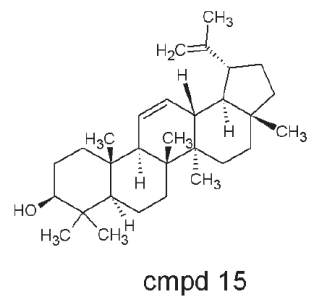

cmpd 15

Figure 4. Biotransformation products of lupeol, caused by $A$. ochraceus and M. rouxii 


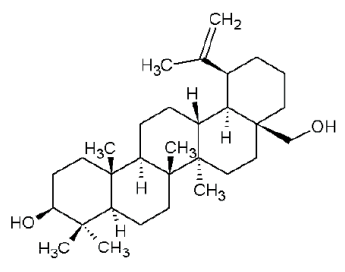

betulin

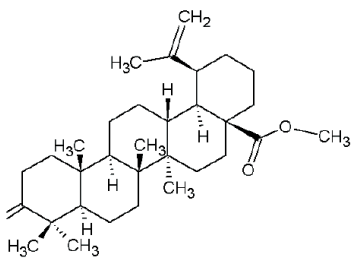

cmpd 9

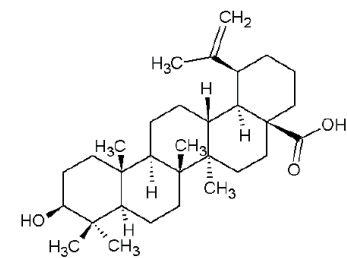

betulinic acid

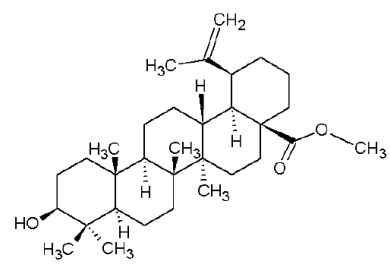

cmpd 10

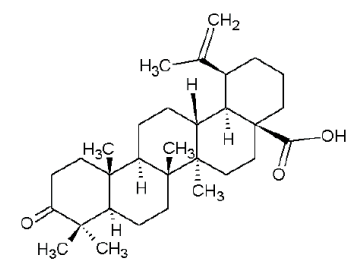

cmpd 8

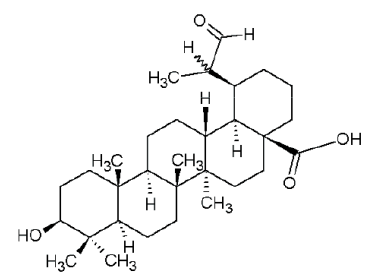

cmpd 11

Figure 5. Biotransformation of betulin and betulinic acid

is a natural pentacyclic triterpenoid compound. It is present in olive-pomace oil, being the main components of the protective wax-like coating of the olive skin. Oleanolic acid displays remarkable pharmacological role due to its antitumor, antibacterial, anti-HIV, anti-inflammatory, antioxidant, and hepatoprotective activity. Biotransformation of oleanolic acid with Rhizomucor miebei yielded a mixture of three metabolites. Chromatography of this mixture on a silica-gel column resulted in recovery of $64 \%$ of the substrate and isolation of the following compounds: queretaroic acid, compound 1 and compound 2 (Fig. 2) (Leipolda et al., 2010).

Ursolic acid, a pentacyclic triterpene, exists in many plant species, especially in some medicinal herbs. It has been reported that ursolic acid exhibits a remarkable spectrum of biological activities, such as antiinflammatory, antiallergic, antibacterial, hepatoprotective and antitumor (Fu et al., 2013).

The Syncephalastrum racemosum converted ursolic acid to five metabolites: compounds 3-7 (Fig. 3) (Fu et al., 2013).

Lupeol has been shown to possess a range of proven biological activities in preventing cancer, coronary and hepatic diseases. It can be very efficiently obtained from bark of some trees (Gallo et al., 2009). Mucor rouxii transformed lupeol to two metabolites, compounds 12 and 13 (Parshikov et al., 2012), although Aspergillus ochraceus also converted lupeol to two metabolites, compound 14 and 15 (Fig. 4) (Carvalho et al., 2010).

Betulinic acid and betulin are bioactive pentacyclic triterpenes. These are the most common triterpenes found in plants together with ursolic and oleanolic acids. They have been isolated from many plants such as birch (Betula spp.). Important pharmacological properties have been described for betulin and betulinic acid. Many studies have confirmed their antitumor, anti-HIV, antiinflammatory, antibacterial, antimalarial, antitrypanosomal and analgesic activity (Baratto et al., 2013). The biotransformation experiments showed production of compounds 8-11 from betulinic acid (Bastos et al., 2007; Baratto et al., 2013). Betulin was converted to betulinic acid using Aspergillus oryzae, Armillaria luteo-virens Sacc, Aspergillus foetidus (Fig. 5) (Chen et al., 2009).

Table 1 shows examples of microbial transformation of triterpenoids and the yields of obtained derivatives which have been already published (Bastos et al., 2007; Chen et al., 2009; Gallo et al., 2009; Carvalho et al., 2010; Leipolda et al., 2010; Parshikov et al., 2012; Baratto et al., 2013; Fu et al., 2013).

\section{CONCLUSIONS}

The use of tissue engineering and cell culture techniques offers the opportunity to optimize the yield of the target compound and to obtain a uniform, high quality product (Mufflera et al., 2011). Microbial transformation of triterpenoids has provided new derivatives that are potentially useful for pharmacological studies. In these biotransformation processes, several reactions that are difficult to achieve by chemical methods have been successfully accomplished. These biotransformations can also be used as in vitro models to predict the metabolism of biologically active triterpenoids (Parra et al., 2009). Plant in vitro production of biologically active metabolites has several advantages because the target substances are produced in cells, tissues or organs that are cultivated in bioreactors under sterile conditions. Moreover, they are completely insulated from adverse environmental factors (Vanisree et al., 2004). Disadvantage of the method is that the amounts of desired metabolites are often lower than the contents in intact plants (Charlwood \& Rhodes, 1990). There are more and more strategies to enhance the yields of secondary metabolites in plant in vitro cultures like media and hormone optimization and the use of various techniques such as immobilization and genetic modification (Dornenburg, 2004; Lee et al., 2004; Pavlov et al., 2007). 
Table 1. Cell cultures applied in synthesis of triterpene derivates

\begin{tabular}{|c|c|c|c|}
\hline $\begin{array}{l}\text { Triterpen com- } \\
\text { pound }\end{array}$ & Cell suspension & Derivate & Yield (\%) \\
\hline \multirow{3}{*}{ Oleanolic acid } & \multirow{3}{*}{$\begin{array}{l}\text { Rhizomucor } \\
\text { miehei }\end{array}$} & $\begin{array}{l}\text { Queretaro- } \\
\text { ic acid }\end{array}$ & 5 \\
\hline & & cmpd 1 & 6 \\
\hline & & cmpd 2 & 4 \\
\hline \multirow{5}{*}{ Ursolic acid } & \multirow{5}{*}{$\begin{array}{l}\text { Syncephalastrum } \\
\text { racemosum }\end{array}$} & cmpd 3 & 13 \\
\hline & & cmpd 4 & 3 \\
\hline & & cmpd 5 & 4 \\
\hline & & cmpd 6 & 3 \\
\hline & & cmpd 7 & 12 \\
\hline \multirow{4}{*}{ Lupeol } & \multirow{2}{*}{ Mucor rouxii } & cmpd 12 & 26 \\
\hline & & cmpd 13 & 16 \\
\hline & \multirow{2}{*}{$\begin{array}{l}\text { Aspergillus ochra- } \\
\text { ceus }\end{array}$} & cmpd 14 & 19 \\
\hline & & cmpd 15 & 11 \\
\hline \multirow{3}{*}{ Betulin } & Aspergillus oryzae & \multirow{3}{*}{$\begin{array}{l}\text { Betulinic } \\
\text { acid }\end{array}$} & 1 \\
\hline & $\begin{array}{l}\text { Armillaria luteo-vi- } \\
\text { rens Sacc QH }\end{array}$ & & 2 \\
\hline & Aspergillus foetidus & & 9 \\
\hline \multirow{6}{*}{ Betulinic acid } & Mycelia sterilia; & cmpd 8 & 3 \\
\hline & \multirow{2}{*}{ Penicillium sp. } & $\mathrm{cmpd} 8$ & 2 \\
\hline & & cmpd 9 & 2 \\
\hline & $\begin{array}{l}\text { Penicillium citreoni- } \\
\text { grum }\end{array}$ & cmpd 10 & 2 \\
\hline & \multirow{2}{*}{ Daucus carota } & cmpd 8 & 7 \\
\hline & & cmpd 11 & 10 \\
\hline
\end{tabular}

\section{REFERENCES}

Barattoa LC, Porsanib MV, Pimentelb IC, Pereira Nettoc AB, Paschked R, Oliveiraa BH (2013) Preparation of betulinic acid derivatives by chemical and biotransformation methods and determination of cytotoxicity against selected cancer cell lines. Eur J Med Chem 68: 121-131.

Bastos DZL, Pimentel IC, de Jesus D, de Oliveira BA (2007) Biotransformation of betulinic and betulonic acids by fungi. Phytochemistry 68: 834-839.

Canter PH, Thomas H, Ernst E (2005) Bringing medicinal plants into cultivation: opportunities and challenges for biotechnology. Trends Biotechnol 23: 180-185.

Carvalho TC, Polizeli AM, Turatti ICC, Severiano ME, Carvalho CE, Ambrósio SR et al.(2010) Screening of filamentous fungi to identify biocatalysts for lupeol biotransformation. Molecules 15: 6140-6151.
Charlwood BV, Rhodes MJC (1990) Secondary products from plant tissue culture. pp. 23-41 Clarendon Press, Oxford.

Chatterjee P, Kouzi SA, Pezzuto JM, Hamann MT (2000) Biotransformation of the antimelanoma agent betulinic acid by Bacillus megaterium ATCC 13368. Appl Environ Microb 66: 3850-3855.

Chen QH, Liu J, Zhang HF, He GQ, Fu ML (2009) The betulinic acid production from betulin through biotransformation by fungi. Enayme Microb Tech 45:175-180.

Cheng ZH, Yu BY, Cordell GA, Qiu SX (2004) Biotransformation of Quinovic Acid Glycosides by Microbes: Direct Conversion of the Ursane to the Oleanane Triterpene Skeleton by Nocardia sp. NRRL 5646. Org Lett 6: 3163-3165.

Dixon RA (2005) Engineering of plant natural product pathways. Curr Opin Plant Biol 8: 329-336.

Dornenburg H (2004) Evaluation of immobilisation effects on metabolic activities and productivity in plant cell processes. Process Biochem 39: 1369-1375.

Dzubak P, Hajduch M, Vydra D, Hustova A, Kvasnica M, Biedermann D (2006) Pharmacological activities of natural triterpenoids and their therapeutic implications. Nat Prod Rep 23: 394-411.

Fu S, Yang J, Cui J, Sun DA (2013) Biotransformation of ursolic acid by Syncephalastrum racemosum CGMCC 3.2500 and anti-HCV activity. Fitoterapia 86: 123-128.

Gallo MBC, Sarachine MJ (2009) Biological activities of lupeol. Int J Biomed Pharm Sci 1: 46-66.

Lee M, Jeong J, Seo J, Shin C, Kim Y, In J et al.(2004) Enhanced triterpene and phytosterol biosynthesis in Panax ginseng overexpressing squalene synthase gene. Plant Cell Physiol 45: 976-984.

Leipolda et al. (2010) Biosynthesis of ursolic acid derivatives by microbial metabolism of ursolic acid with Nocardia sp. strains - Proposal of new biosynthetic pathways Roland Ulber. Process Biochem 45: 1043-1051.

Lohr M, Schwender J, Polle JEW (2012) Isoprenoid biosynthesis in eucaryotic phototrophs: A spotlight on algae. Plant Sci 185-186: 9-22.

Mufflera K, Leipolda D, Schellera MC, Haasb C, Steingroewerb J, Bleyb T, Neuhausc HE, Miratad MA, Schraderd J, Ulbera R (2011) Biotransformation of triterpenes. Proc Biochem 46: 1-15.

Marienhagen J, Bott M (2013) Metabolic engineering of microorganisms for the synthesis of plant natural products. I Biotech 163: 166178.

Parra A, Rivas F, Garcia-Granados A, Martinez A (2009) Microbial transformation of triterpenoids. Mini-Rev Org Chem 6: 307-320.

Parshikov IA, Netrusov IA, Sutherland JB (2012) Microbial transformation of antimalarial terpenoids. Biotech Adv 30: 1516-1523.

Pavlov A, Georgiev M, Bley T (2007) Batch and fed-batch production of betalains by Red Beet (Beta vulgaris) hairy roots in a bubble column reactor. $Z$ Naturforsch $C$ 62: 439-446.

Qian LW, Zhang J, Liu JH, Yu BY (2009) Direct microbial-catalyzed asymmetric [alpha]-hydroxylation of betulonic acid by Nocardia sp. NRRL 5646. Tetrahedron Lett 50: 2193-2195.

Rao SR, Ravishankar GA (2002) Plant cell cultures: chemical factories of secondary metabolites. Biotechnol Adv 20: 101-153.

Vanisree M, Lee CY, Lo SF, Nalawade SM, Lin CY, Tsay HS (2004) Studies on the production of some important secondary metabolites from medicinal plant tissue cultures. Bot Bull Acad Sin 42: 1-22.

Waller GR (1970) Metabolism of plant terpenoids. Prog Chem Fats Other Lipids 10: 153-212.

Zhanga J, Chenga ZH, Yua BY, Cordellb GA, Qiuc SX (2005) Novel biotransformation of pentacyclic triterpenoid acids by Nocardia sp. NRRL 5646. Tetrahedron Lett 46: 2337-2340 